\title{
Novel Structure Function for Photon Fragmentation into a $\Lambda$ Hyperon and Transverse $\Lambda$ Polarization in Unpolarized Electron-Positron Annihilation
}

\author{
Wei Lu \\ CCAST (World Laboratory), P.O. Box 8730, Beijing 100080, China \\ and Institute of High Energy Physics, P.O. Box 918(4), Beijing 100039, Chind
}

\begin{abstract}
The possibility is examined for the inclusive $\Lambda$ in unpolarized electron-positron annihilation to be transversely polarized. Due to final-state interactions, there exists a novel structure function $\hat{F}\left(z, Q^{2}\right)$ for the inclusive $\Lambda$ hyperon (or any other baryons) production from the unpolarized time-like photon fragmentation, which makes contribution to the transverse $\Lambda$ polarization in the unpolarized electron-positron annihilation.
\end{abstract}

PACS numbers: 13.88.+e, 13.65.+i

Typeset Using REVTEX

${ }^{*}$ Mailing address 
During the past two decades, quantum chromodynamics (QCD) has been established gradually as the most prospective candidate for the underlying strong-interaction theory. In interpreting various spin asymmetries and final-state transverse polarization phenomena, however, it is not so successful. Early in 1970's, for example, people [1] at Fermilab observed large $\Lambda$ polarization perpendicular to the production plane in unpolarized $\mathrm{p}-\mathrm{B}_{\mathrm{e}}$ experiments. Until now, no completely QCD-based scenario has been successfully established to explain such seemingly spectacular phenomena.

By the standard helicity amplitude analysis [2], the transverse polarization of inclusive fermion is proportional to the interference of its two helicity amplitudes. In a massless vector or axial-vector theory, if there is no novel physical mechanism, there exists no transverse polarization. As the effects of particle masses are included, intuitively, the mass-induced polarization is to be proportional to the mass parameter. It is based on this fact that Kane, Pumplin and Repko [3] pointed out that hard partonic scatterings cannot take the whole responsibility for the large transverse $\Lambda$ polarization in hadronic processes. In general, the transverse quark polarization due to hard partonic subprocesses assumes the following asymptotic behavior:

$$
P_{q} \propto \frac{\alpha_{s} m_{q}}{Q}
$$

where $Q$ is the scale of hard scatterings. In the parton model, the parton fragments into hadrons independently, so the transverse polarization of inclusive baryon can only come from that of partons responsible for its production. Since so, the search is in need for other possible resources of the large transverse $\Lambda$ polarization.

For the inclusive fermion production, if we denote the momenta of the beam and inclusive fermion by $\mathbf{p}$ and $\mathbf{k}$ respectively, the transverse fermion polarization is proportional to the expectation value $<\mathbf{s} \cdot(\mathbf{p} \times \mathbf{k})>$. Despite $\mathbf{s} \cdot(\mathbf{p} \times \mathbf{k})$ is time-reversal odd, final-state interactions may make contributions to the transverse polarization [4]. Therefore, it is an interesting theme to examine how well final-state interactions contribute to the transverse 
polarization of inclusive observed $\Lambda$ hyperons in fixed-target experiments. At present, it is not an easy job because of the complexities of hadronic processes.

Alternatively, we investigate in this Brief Report the possibility of studying the effects of final-state interactions in the process

$$
e^{-}(p)+e^{+}(q-p) \rightarrow \Lambda(k, s)+X
$$

where $s^{\mu}$ is the $\Lambda$ spin vector and normalized as $s \cdot s=1$ for a pure state. Naively, since the initial-state particles are unpolarized, the inclusive $\Lambda$ production seems to have no spin weights. Owing to the interactions between the final-state particles, however, there do exist possibilities for the inclusively detected $\Lambda$ 's to be transversely polarized, as we will show in the coming discussion.

We choose to work in the energy region far below the $Z$-resonance. In such a case, electron-positron annihilation proceeds predominantly via the single-photon channel. Correspondingly, its differential cross section may be written as

$$
\mathrm{d} \sigma=\frac{1}{2 Q}\left(\frac{4 \pi \alpha}{Q^{2}}\right)^{2}(4 \pi) L_{\mu \nu} \tilde{W}^{\mu \nu} \frac{\mathrm{d}^{3} k}{(2 \pi)^{3} 2 E}
$$

where $Q=\sqrt{q^{2}}$ is the c.m. energy, $L_{\mu \nu}$ the lepton tensor, and $\tilde{W}_{\mu \nu}$ the photon fragmentation tensor for the inclusive $\Lambda$ production. We isolate the electromagnetic coupling constant $e^{2}=$ $4 \pi \alpha$ for each tensor in the sense that the electromagnetic current is defined as $j_{\mu}=Q_{f} \bar{\psi} \gamma_{\mu} \psi$. In addition, the electron mass is ignored.

Now we dwell on the Lorentz decomposition of photon fragmentation tensor $\tilde{W}_{\mu \nu}$. In the case of inclusive baryon production, it is considered usually without spin information. In this Brief Report, we concentrate ourselves on the case of measuring the spin of inclusively produced $\Lambda$ hyperon.

The photon fragmentation tensor for the inclusive $\Lambda$ production is defined as

$$
\tilde{W}_{\mu \nu}(k, q, s)=\frac{1}{4 \pi} \int \mathrm{d}^{4} x e^{i q \cdot x}<0\left|j_{\mu}^{\dagger}(0)\right| \Lambda(k, s), X>_{\text {out }} \text { out }<\Lambda(k, s), X\left|j_{\nu}(x)\right| 0>
$$


Throughout our work, the summation over the unobserved system $X$ is understood. The general Lorentz decomposition of $\tilde{W}_{\mu \nu}(q, k, s)$ is subjected to parity conservation, time reversal invariance and current conservation. In addition, the hermiticity of $j_{\mu}$ leads to

$$
\tilde{W}_{\mu \nu}(k, q, s)=\tilde{W}_{\nu \mu}^{*}(k, q, s)
$$

In obtaining the general expression for $\tilde{W}_{\mu \nu}(q, k, s)$, the Lorentz vectors and tensors to be employed are $k_{\alpha}, q_{\alpha}, s_{\alpha}, g_{\alpha \beta}$ and $\varepsilon_{\alpha \beta \gamma \delta}$.

Let us imagine a process of deeply inelastic scattering for the unpolarized electron beam and polarized $\bar{\Lambda}$ target. Then, process (2) can be related to it by reversing the momenta and spin vector of position and $\Lambda$ particle. Intuitively, the hadronic tensor for $\bar{\Lambda}$ and photon fragmentation tensor for the inclusive $\Lambda$ production should bear the same Lorentz structures, since both two are subjected to the same symmetries. The hadronic tensor of spin-half targets has been well-known [5]. By analogy, the decomposition of $\tilde{W}_{\mu \nu}(k, q, s)$ contains at least the following combinations:

$$
-g_{\mu \nu}+\frac{q_{\mu} q_{\nu}}{q^{2}},\left(k_{\mu}-\frac{k \cdot q}{q^{2}} q_{\mu}\right)\left(k_{\nu}-\frac{k \cdot q}{q^{2}} q_{\nu}\right), i \varepsilon_{\mu \nu \lambda \sigma} q^{\lambda} s^{\sigma}, i \varepsilon_{\mu \nu \lambda \sigma} q^{\lambda}\left(s^{\sigma}-\frac{s \cdot q}{k \cdot q} k^{\sigma}\right) .
$$

In the case of unpolarized electron-positron annihilation, parity conservation determines that the inclusive $\Lambda$ can be transversely polarized only. Correspondingly, $s \cdot q=0$, and the last two of the combinations displayed above are degenerate. However, if initial-state particles are polarized, $s \cdot q$ vanishes no longer. Although our discussions will be concentrated on the unpolarized annihilation, formally we still distinguish the above two spin-dependent terms.

Our findings to be presented are based upon the following observation. That is, the hadronic tensor is built on a single-particle state only, while fragmentation tensor involves multi-particle final states. Such a difference causes their distinct behaviors under time reversal transformation, because under time reversal transformation an out-state is changed into its corresponding in-state. Unless it is a single-particle state, an out-state differs from its corresponding in-state by a unitary transformation S [6]. Generally, the S operator (matrix) can be written as 


$$
\left|>_{\text {in }}=S\right|>_{\text {out }}, \quad \text { with } S=1+i T
$$

where $T$ is the transition operator (matrix).

We examine how the fragmentation tensor behaves under $P T$ transformation instead of individual transformations $P$ and $T$. Under $P T$ transformation, spin vector changes sign while momentum does not, correspondingly it is convenient to study spin asymmetries. In our case,

$$
\begin{aligned}
\tilde{W}_{\mu \nu}(k, q, s) & =\frac{1}{4 \pi} \int \mathrm{d}^{4} x e^{i q \cdot x}\left[<0\left|j_{\mu}^{\dagger}(0)\right| \Lambda(k,-s), X>_{\text {in }} \text { in }<\Lambda(k,-s), X\left|j_{\nu}(-x)\right| 0>\right]^{*} \\
& =\left[\frac{1}{4 \pi} \int \mathrm{d}^{4} x e^{i q \cdot x}<0\left|j_{\mu}^{\dagger}(0) S\right| \Lambda(k,-s), X>_{\text {out }} \text { out }<\Lambda(k,-s), X\left|S^{\dagger} j_{\nu}(x)\right| 0>\right]^{*} \\
& =\left[\frac{1}{4 \pi} \int \mathrm{d}^{4} x e^{i q \cdot x}<0\left|j_{\mu}^{\dagger}(0)\right| \Lambda(k,-s), X>_{\text {out }} \text { out }<\Lambda(k,-s), X\left|j_{\nu}(x)\right| 0>\right]^{*}+\cdots \\
& =\tilde{W}_{\mu \nu}^{*}(k, q,-s)+\cdots .
\end{aligned}
$$

This is completely different from the case of deeply inelastic scattering, in which one can derive $W_{\mu \nu}(q, p, s)=W_{\mu \nu}^{*}(q, p,-s)$. The terms denoted by ellipses in Eq. (7) are not subjected to the constraint $\tilde{W}_{\mu \nu}(q, p, s)=\tilde{W}_{\mu \nu}^{*}(q, p,-s)$. As a consequence, the general expression of $\tilde{W}_{\mu \nu}$ may contain other terms rather than those that have counterparts in $W_{\mu \nu}(q, p, s)$.

Physically, these novel terms are due to the interactions between final-state particles $[7,8]$. To see this, let us examine the following term that was suppressed in Eq. (7):

$$
-\frac{i}{4 \pi}\left[\int \mathrm{d}^{4} x e^{i q \cdot x}<0\left|j_{\mu}^{\dagger}(0) T\right| \Lambda(k,-s), X>_{\text {out }} \text { out }<\Lambda(k,-s), X\left|j_{\nu}(x)\right| 0>\right]^{*}
$$

Making use of the completeness of final states, we can insert $\int \mathrm{d} \Gamma \mid$ f.s. $>_{\text {out }}$ out $<$ f.s. $\mid=1$ before the transition operator $T$, where $\mid$ f.s. $>$ and $\mathrm{d} \Gamma$ stand for the final state and its phase space elements, and the integration includes the summation over all the discrete degrees 
of freedom. Then we have the following physical picture: A time-like photon decays first into other possible intermediate physical states, which then transit via interactions to the inclusively detected final state. It is such final-state scatterings that make the relative phase between an in-state and its corresponding out-state.

Subject to the constraints from all symmetries, the novel terms arising from final-state interactions can only be of the Lorentz structure

$$
\left[\left(k_{\mu}-\frac{k \cdot q}{q^{2}} q_{\mu}\right) \varepsilon_{\nu \alpha \beta \gamma} k^{\alpha} q^{\beta} s^{\gamma}+\left(k_{\nu}-\frac{k \cdot q}{q^{2}} q_{\nu}\right) \varepsilon_{\mu \alpha \beta \gamma} k^{\alpha} q^{\beta} s^{\gamma}\right]
$$

Thus, the full decomposition of photon fragmentation tensor reads

$$
\begin{aligned}
\tilde{W}_{\mu \nu}(k, q, s) & =\frac{1}{2}\left[\left(-g_{\mu \nu}+\frac{q_{\mu} q_{\nu}}{q^{2}}\right) \tilde{F}_{1}+\left(k_{\mu}-\frac{k \cdot q}{q^{2}} q_{\mu}\right)\left(k_{\nu}-\frac{k \cdot q}{q^{2}} q_{\nu}\right) \frac{\tilde{F}_{2}}{k \cdot q}\right] \\
& +i M_{\Lambda} \varepsilon_{\mu \nu \lambda \sigma} q^{\lambda} s^{\sigma} \frac{\tilde{g}_{1}}{k \cdot q}+i M_{\Lambda} \varepsilon_{\mu \nu \lambda \sigma} q^{\lambda}\left(s^{\sigma}-\frac{s \cdot q}{k \cdot q} k^{\sigma}\right) \frac{\tilde{g}_{2}}{k \cdot q} \\
& +M_{\Lambda}\left[\left(k_{\mu}-\frac{q \cdot k}{q^{2}} q_{\mu}\right) \varepsilon_{\nu \alpha \beta \gamma} k^{\alpha} q^{\beta} s^{\gamma}+\left(k_{\nu}-\frac{q \cdot k}{q^{2}} q_{\nu}\right) \varepsilon_{\mu \alpha \beta \gamma} k^{\alpha} q^{\beta} s^{\gamma}\right] \frac{\hat{F}}{(k \cdot q)^{2}}
\end{aligned}
$$

The factor $\frac{1}{2}$ in the spin-independent terms implies the fact that we must perform spin summation if we do not measure the $\Lambda$ spin. $\hat{F}$ is a novel structure function arising from the final-state interactions. We have borrowed the labels from the expression of hadronic tensor for the other four structure functions and added to them a tilde. $\tilde{F}_{1}$ and $\tilde{F}_{2}$ are spin independent, while $\tilde{g}_{1}, \tilde{g}_{2}$ and $\hat{F}$ are spin depedent. By the hermiticity of electromagnetic current, all five fragmentation structure functions are real. In addition, all of them depend on $z=\frac{2 k \cdot q}{q^{2}}$ and $Q^{2}=q^{2}$.

The novel structure function $\hat{F}$ is closely related to the transverse polarization of the inclusive $\Lambda$ hyperon in the initial-state unpolarized electron-positron annihilation, which is defined by

$$
P_{\Lambda}=\frac{\mathrm{d} \sigma\left(s_{\uparrow}\right)-\mathrm{d} \sigma\left(s_{\uparrow}\right)}{\mathrm{d} \sigma\left(s_{\downarrow}\right)+\mathrm{d} \sigma\left(s_{\downarrow}\right)}
$$


Recall that when the initial-state electron and positron are unpolarized, lepton tensor is both real and symmetric under $\mu \leftrightarrow \nu$. Therefore, only the novel term in Eq. (8) contributes to the $\Lambda$-spin dependence of cross section. Schematically, we have

$$
P_{\Lambda}=\frac{\left.L_{\mu \nu} \tilde{W}^{\mu \nu}\left(q, k, s_{\uparrow}\right)\right|_{\text {novel }}}{\left.L_{\mu \nu} \tilde{W}^{\mu \nu}\left(q, k, s_{\uparrow}\right)\right|_{\text {spin independent }}} .
$$

After a little algebra, we obtain

$$
P_{\Lambda}=\frac{2 M_{\Lambda} \sin \theta \cos \theta \hat{F}}{Q\left(1+\frac{M_{\Lambda}^{2}}{\mathbf{k}^{2}}\right) \tilde{F}_{1}+\frac{1}{2} \sqrt{\mathbf{k}^{2}+M_{\Lambda}^{2}} \sin ^{2} \theta \tilde{F}_{2}}, \text { with } \theta=\angle(\mathbf{p}, \mathbf{k}) \text {. }
$$

The structure functions $\tilde{F}_{1}$ and $\tilde{F}_{2}$ are associated with the inclusive $\Lambda$ hyperon production without measuring its spin. If one works with the parton model, $\tilde{F}_{1}$ is related to $\tilde{F}_{2}$ by $2 \tilde{F}_{1}+z \tilde{F}_{2}=0$. At present, the $\Lambda$ production study based on the BES data at BEPC is under the way [9]. It can be anticipated that the measurements of $\tilde{F}_{1}$ and $\tilde{F}_{2}$ are realized in the near future.

As we have identified, $\hat{F}$ incorporates the effects of final-state interactions on the transverse $\Lambda$ polarization in unpolarized electron-positron annihilation. Since it evolves multiple interactions and the hadronic parton interactions become more and more dominant in the high-energy case, the effects of $\hat{F}$ are power-suppressed, or so-called higher-twist effects. In fact, all transverse spin asymmetries are twist-three effects [10]. It is known that in the moderate kinematical region some higher twist effects can become relatively large [11]. In the energy region in which BEPC, TRISTAN and the proposed Beauty and Tau-Charm Factories lie, it is quite probable that final-state interactions are not shadowed by hard partonic physics. Here, we suggest measuring the transverse polarization of the inclusively produced $\Lambda$ hyperon in the initial-state unpolarized electron-positron annihilation. Process (2) is an ideal place to examine how well final-state interactions contribute to the transverse $\Lambda$ polarization. Since the final-state interactions are widely existent in any inclusively detected particle system, we may anticipate the measurement of transverse $\Lambda$ polarization in unpolarized electron-positron annihilation will help our understanding of transverse hyperon 
polarization phenomena. Whether positive or negative, the results of the suggested experiment will be enlightening. On the one hand, if a large transverse polarization is detected, then we may expect that final-state interactions play a significant role in the transverse $\Lambda$ polarization in fixed-target processes, although these experiments are done at somewhat different energy scales. On the other hand, if there is little transverse polarization in process (2), we are supposed to search for other possible resources for the large transverse $\Lambda$ polarization phenomena.

In conclusion, we examined the general Lorentz decomposition of photon fragmentation tensor for the inclusive $\Lambda$ hyperon production. By emphasizing the difference between the fragmentation tensor and hadronic tensor in deeply inelastic scattering, we point out that there exists a novel term in the general expression of the former, which is associated with the possible transverse $\Lambda$ polarization in unpolarized electron-positron annihilation. Physically, such a transverse polarization is due to the interactions between the final-state particles. We suggest measuring the transverse polarization in process (2) so as to obtain some indications how well final-state interactions contribute to the large transverse $\Lambda$ polarization in fixedtarget experiments.

\section{ACKNOWLEDGMENTS}

The author is indebted to T. Huang, X.Q. Li, C.W. Luo and Y.G. Xie for encouraging discussions. 


\section{REFERENCES}

[1] G. Bunce et al., Phys. Rev. Lett. 36, 1113 (1976)

[2] M. Jacob and G.C. Wick, Ann. Phys. 7, 404 (1959)

[3] G.L. Kane, J. Pumpkin, and W. Repko, Phys. Rev. Lett. 41, 1689 (1978)

[4] S. Gasiorowicz, Elementary Particle Physics (Wiley, New York, 1966).

[5] B.L. Ioffe, V.A. Khoze and L.N. Lipatov, Hard Processes (Elsivier, Amsterdam, 1984), Vol. 1.

[6] J.D. Bjorken and S.D. Drell, Relativistic Quantum Fields (McGraw-Hill, New York, 1965).

[7] R.L. Jaffe and X. Ji, Phys. Rev. Lett. 71, 2547 (1993)

[8] X. Ji, Phys. Rev. D 49, 114 (1994).

[9] Y.G. Xie, private communication.

[10] J. Qiu and G. Sterman, Polarized Collider Workshop, edited by J.C. Collins, S.F. Heppelman, and R.W. Robinett (American Institute of Physics, New York, 1990) p. 249; Nucl. Phys. B 353, 137 (1991).

[11] J. Qiu and G. Sterman, Phys. Rev. Lett. 67, 2264 (1991); Nucl. Phys. B 378, 52 (1992). 